\title{
The Cyclic Behavior of Mountain Gravity Waves Generated by Flow over Topography*
}

\author{
Ziliang Li", Changji Chen, Jinqing Liu \\ Department of Marine Meteorology, Physical Oceanography Laboratory, Ocean University of China, Qingdao, China \\ Email: "liziliang@ouc.edu.cn
}

Received March 7, 2013; revised April 9, 2013; accepted May 6, 2013

Copyright (C) 2013 Ziliang Li et al. This is an open access article distributed under the Creative Commons Attribution License, which permits unrestricted use, distribution, and reproduction in any medium, provided the original work is properly cited.

\begin{abstract}
The cyclic behavior of lee wave systems, generated by stratified flow over mountains is investigated by the Advanced Regional Prediction System (ARPS) model. The results show that, surface friction has a direct impact upon the number and timing of mountain gravity waves cycle generation. Cyclic generation of mountain lee waves and down-slope winds was found to be extremely sensitive to the magnitude of the surface drag coefficient, where mountain waves amplitude and intensity varies with the magnitude of the drag coefficient, and the interaction of mountain waves and boundary layer process determinates the wave characteristics. For the typical drag $C_{d}=10^{-3}$, surface friction promotes the formation of the stationary mountain lee waves and hydraulic jump, especially, promotes boundary layer separation, the generation of low-level turbulent zones and rotor circulation or reversal flow within boundary layer. When drag coefficient becomes $C_{d}=10^{-4}$, lee waves remain steady states and the first evolution cycle maintains much longer than that of $C_{d}=$ $10^{-3}$. In the case of the highest drag coefficient $C_{d}=10^{-2}$, surface friction suppresses wave breaking and the onset of hydraulic jump, and reduces greatly the amplitude and intensity of lee waves and down slope wind.
\end{abstract}

Keywords: Lee Waves; Downslope Wind; Surface Friction; Cyclic; Numerical Simulation

\section{Introduction}

Although mountain lee waves have been studied extensively from theoretical and numerical modeling studies through to laboratory and observational experiments [1-5], the influence of surface friction effects on these waves has received little attention until recently, due partly to the difficult of introducing surface friction in theoretical models. Recent measurements and numerical simulation results suggest that processes that have received relatively little emphasis previously, such as surface friction, may be important for lee wave generation and development [6-10]. Richard [11] suggested that simulations with friction were qualitatively more realistic than simulations without surface friction, that the genesis of strong winds on the lee slope was delayed when surface friction was included, and that the downstream propagation of a mid-troposphere jump-like feature was impeded in the absence of friction. Olafsson and Bougeault $[12,13]$ showed that surface friction plays an active role in suppressing wave breaking for the negative impact of

\footnotetext{
${ }^{*}$ This work was supported by the National Natural Science Foundation of China (Grant No. 41176005).

"Corresponding author.
}

surface friction on wave activity. Doyle and Durran [14] demonstrate that the realistic rotors appear to develop only in the presence of surface friction by using numeric simulations with free slip and no slip lower boundary layer conditions. Peng and Thompson [15] investigated the effect of boundary layer including surface friction on flow over mountains, and they suggested that the reduction in mountain wave amplitude in the presence of surface friction is due to the reduction in the slope of the boundary layer height as compared to the terrain height. A wide body of studies has described the atmospheric gravity wave dynamics, which play a significant role in energy transport and hazard weather forecast [16-18].

These analysis, in general, conclude that surface friction reduces or suppresses wave breaking, and reduces the amplitude and intensity of lee waves, however, surface friction promotes the boundary layer separation, the generation of low-level turbulent zones and rotor circulation or reversal flow. On the other hand, meteorological observations and experimental data seem to suggest otherwise. It is thus of interest to study the effect of surface friction on flow over topography. In the present study, we seek to identify more specifically a mechanism that 
may provide an explanation for the effect of surface friction on lee waves. The numerical model used here is the Advanced Regional Prediction System (ARPS), described in Section 2. Section 3 is devoted to the numeric simulations of flow over topography, exploring the interaction of the flow with topography including friction dissipation. Our conclusions are presented in Section 4.

\section{Model Description}

The simulation experiments are conducted in there using the Version 5.0.0.0IHOP5 of the Advanced Regional Prediction System (ARPS), ARPS is a three-dimensional, non-hydrostatic model developed for storm scale numerical weather prediction in the University of Oklahoma. For the simulations discussed herein, considering the Coriolis force is set to zero and the atmosphere is assumed to be no surface sensible or latent heat fluxes exist, and other physical processes, such as the radiation model and the soil model are not included for the sake of simplicity.

The computational domain consists of 263 grid points with $1 \mathrm{~km}$ horizontal grid spacing in the horizontal direction and 83 layers in the vertical with the Rayleigh damping applied over the top half depth. The vertical grid spacing varies smoothly from $0.05 \mathrm{~km}$ at the ground stretching to $0.25 \mathrm{~km}$ near the top of the domain via

$$
\left\{\begin{array}{l}
\Delta z(i)=\Delta z_{m}+\frac{\Delta z_{\min }-\Delta z_{m}}{\tanh (2 \alpha)} \tanh \left[\frac{2 \alpha}{1-a}(i-a)\right] \\
a=\frac{1+(n z-3)}{2} \text { for } i=1,2, \ldots, n z-3
\end{array}\right.
$$

where $\Delta z_{m}$ is the average grid spacing $0.25 \mathrm{~km}, \alpha$ is a tuning parameter set to unity, and $n z$ is the number of the grid points in the vertical. In addition, radiation boundary condition is applied in east-west direction, while periodic boundaries are used in north-south direction, and the big time step and small time step are $2 \mathrm{~s}$ and $0.5 \mathrm{~s}$, respectively.

Surface friction is included in the ARPS surface physics package, which includes parameterization of surface fluxes as momentum stresses at the lower boundary:

$$
\begin{gathered}
-\left.\tau_{13}\right|_{\text {surface }}=\bar{\rho} C_{d}[|V| u-|\bar{V}| \bar{u}] \\
-\left.\tau_{23}\right|_{\text {surface }}=\bar{\rho} C_{d}[|V| v-|\bar{V}| \bar{v}]
\end{gathered}
$$

where $\tau_{13}$ is the momentum stress on the constant $x$ plane and $\tau_{23}$ is the momentum stress on a constant $y$ plane in the vertical direction, $C_{d}$ is the non-dimensional drag coefficient, $|V|$ is wind speed, and over-bars represent the base state. And the parameterization of the vertical turbulent diffusion follows the 1.5-order turbulent kineticenergy closure.

The basic state velocity $U=20 \mathrm{~ms}^{-1}$ and static stability
$N^{2}=0.01 \mathrm{~s}^{-1}$, and the mountain profile used in here is the bell-shaped mountains, given as

$$
h(x, y)=\frac{h_{\max } a^{2}}{1+\left(x-x_{0}\right)^{2}}
$$

where $h_{\max }$ is the maximum height of mountain, the half width of mountain $a=10 \mathrm{~km}$, and the mountain center location in horizontal direction $x_{0}=72 \mathrm{~km}$.

\section{Results and Interpretations}

We first consider the atmosphere to be uniform with height far upstream of the mountain to identify the evolution of lee wave systems. In the absence of surface friction, vertically propagating gravity waves typically form when the maximum height of mountain $h_{\max }=0.5$ $\mathrm{km}$. However, when the maximum height of mountain increases to $h_{\max }=2 \mathrm{~km}$, then the airflow may be blocked on the upwind slope and the trapped lee waves form on the downstream lee side, accompanied by the hydraulic jumps.

When surface friction is activated, the flow pattern in the lower atmosphere becomes quite different from that of free slip case. When choosing $h_{\max }=0.5 \mathrm{~km}$, it is extremely sensitive to the distance $Z_{h}$ between the surface ( $u=v=w=0$ ) and the first model grid point layer. Such as $Z_{h}=25 \mathrm{~m}$, the influence of surface friction on the vertical propagating waves is relatively minor compared with the free-slip case. However, if $Z_{h}$ increases from 25 $\mathrm{m}$ to $125 \mathrm{~m}$, due to the deeper depth of the positive vertical wind shear in the boundary layer, surface friction is known to reduce the tendency toward wave breaking, apart from the general reduction of the near surface winds speed, these results are consistent with previous work $[6,11,12,15]$, especially under no slip conditions.

However, if choosing $h_{\max }=2 \mathrm{~km}$, surface friction has a direct impact upon the number and timing of lee wave cycles. In the first cycle of wave evolutions, the results are consistent with the simulations of Richard [11] and others work, but involved in cyclic generation of lee wave systems in our run, the results are different from others for the regeneration of lee waves in the second cycle. Most significantly, the effect of surface friction on lee wave amplitude and intensity varies with the magnitude of drag coefficient, especially, cyclic generation of lee waves and down slope winds in our runs was found to be extremely sensitive to the magnitude of drag coefficient. In addition, surface friction promotes the boundary layer separation and the forming of rotors within the boundary layer.

For the typically surface friction drag coefficient $C_{d}=$ $10^{-3}$, the cyclic regeneration of lee wave systems is apparent and allows the wave systems to undergo one transition of two cycles in the whole integration of 144,000 s, 
in which lee wave system begin to dissipate and complete one cycle after the integration of almost 26,000 s and they begin to regenerate and intensify again after integration of almost $36,000 \mathrm{~s}$, the interaction of lee waves and rotors circulation finally determine the flow characters. Firstly, we can see that from Figures 1 and 2, in the first cycle stage, lee waves intensify and low level closed rotor circulation form after integration of almost 12,000 s,
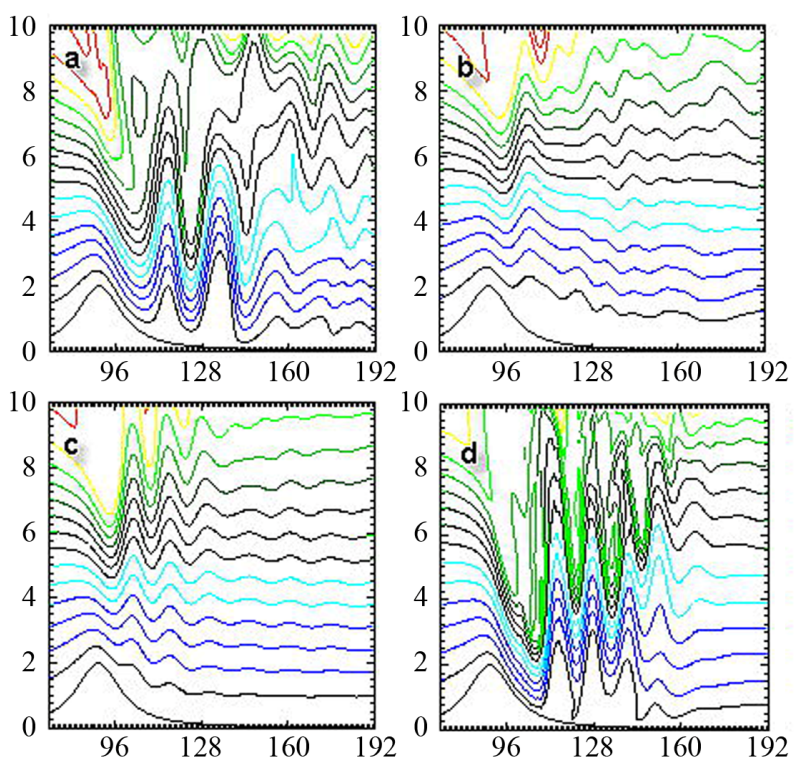

Figure 1. Potential temperature $(\mathrm{K})$ for the simulation of flow over a $2 \mathrm{~km}$ mountain with surface friction of $C_{d}=10^{-3}$, after integration of (a) 12,000 s; (b) 26,000 s; (c) 36,000 s; (d) 76,000 s.
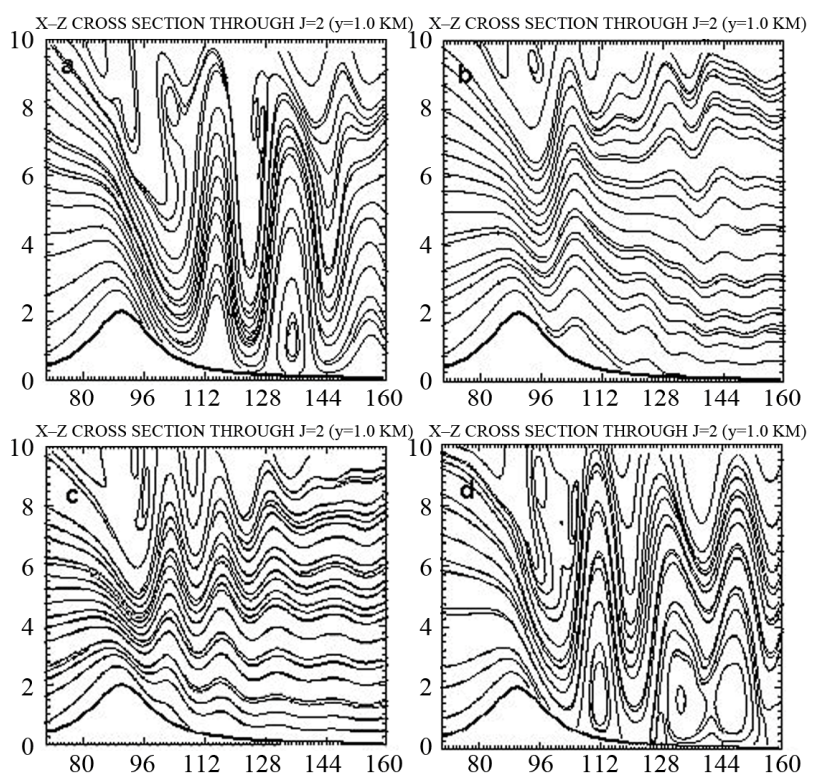

Figure 2. Streamlines for the simulation of flow over a $2 \mathbf{~ k m}$ mountain with surface friction of $C_{d}=10^{-3}$, after integration of (a) 12,000 s; (b) 26,000 s; (c) 36,000 s; (d) 76,000 s. where the low level rotors and rotor circulation play a negative role in the amplitude and intensity of lee waves, and lee waves vanish for surface friction after integrating of almost $26,000 \mathrm{~s}$. In the second cycle stage, lee waves begin to occur in the middle and upper atmosphere after integrating of 36,000 s. As time increases, then hydraulic jump, large amplitude lee waves, lee vortex, organized rotor and rotor circulation regenerate after integrating of 76,000 s. Secondly, surface friction reduces the magnitude of the down slope wind, suppresses the amplitude and intensity of lee waves in the first cycle. However, in the second cycle, the influences of surface friction upon the magnitude of the down slope wind appears to be relatively minor, and surface frictions have a minimal impact upon the structures of lee waves in the middle and upper above the boundary layer. Such as, the maximum cross ridge speed attains $80.2 \mathrm{~ms}^{-1}$ in free-slip condition, but for the drag coefficient $C_{d}=10^{-3}$, it almost attains the value $57.8 \mathrm{~ms}^{-1}$ in the first cycle and almost the same value $78 \mathrm{~ms}^{-1}$ in the second cycle, respectively.

When the drag coefficient is smaller than the typically value $C_{d}=10^{-3}$, that is, the drag coefficient reduced by $C_{d}=10^{-4}$, except low level rotors and rotor circulations are established in the first cycle, compared with the freeslip case, the influences of surface friction upon the magnitude of lee waves and down slope wind appears to be relatively minor, but the regeneration of lee waves is delayed greatly compared with the case $C_{d}=10^{-3}$. The potential temperature, streamline and horizontal velocity given by Figures $\mathbf{3}$ and $\mathbf{4}$ show that, in the first cycle, hydraulic jump lee waves and lee vortex occur above the boundary layer with lee rotors within the boundary layer,
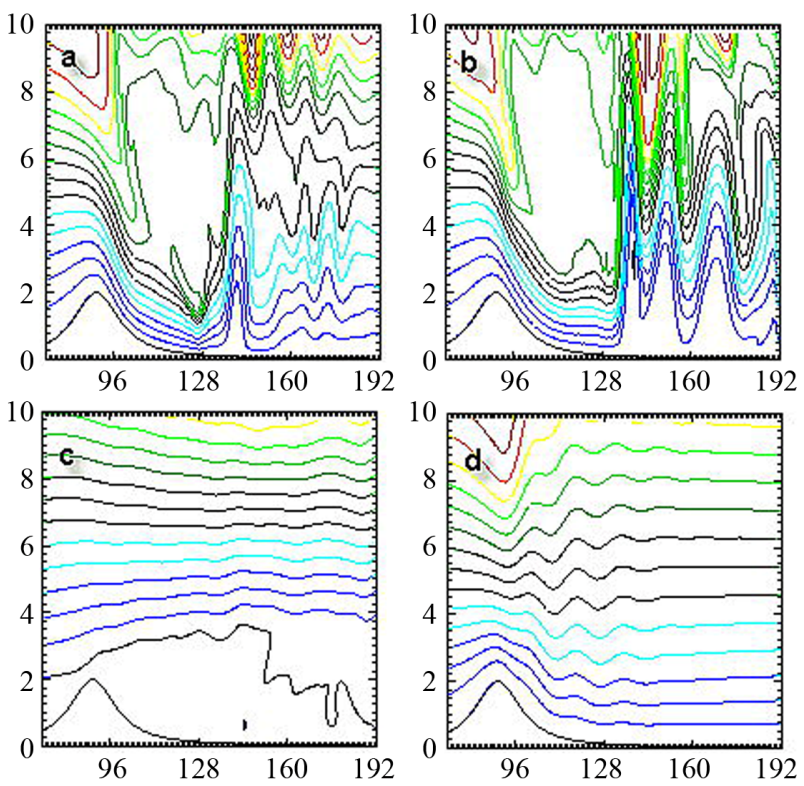

Figure 3. Same as Figure 2 but for the case with surface friction of $C_{d}=10^{-4}$. 

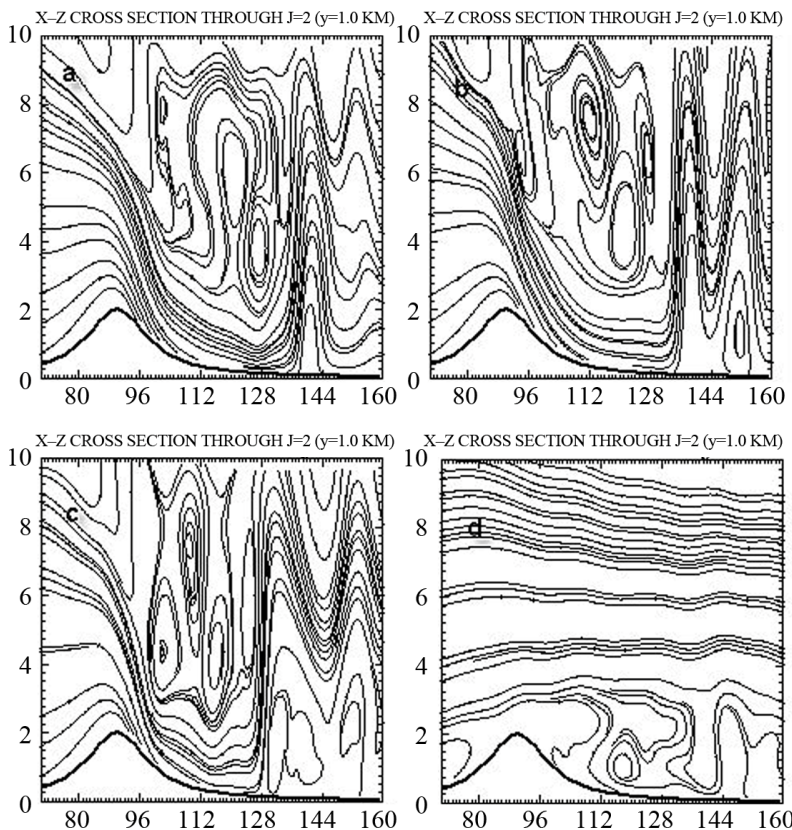

Figure 4. Same as Figure 2 but for the case with surface friction of $C_{d}=10^{-2}$.

and the maximum cross ridge speed is attains $82.2 \mathrm{~ms}^{-1}$ in the first cycle. However, it is quite different from the free-slip case and the case $C_{d}=10^{-3}$, lee wave systems have dissipated for surface friction after integrating of $36,000 \mathrm{~s}$, and they will begin to regenerate slowly again after integrating of $126,000 \mathrm{~s}$, the time interval between the two cycles is apparent elongated, and the life span of the first cycle increases nearly 18,000 $\mathrm{s}$ and the second cycle is delayed nearly 76,000 s compared with the case $C_{d}=10^{-3}$. This sensitivity of lee wave to drag coefficient suggests that there is an optimum value of surface friction (approximately $C_{d}=10^{-4}$ ) that maximizes the strength of the mountain wave systems for this particular simulation.

When surface friction drag coefficient is increased, for the case of $C_{d}=10^{-2}$, surface friction suppresses wave breaking and the onset of hydraulic jump, and reduces greatly the amplitude and intensity of lee waves and down slope wind, wherein the lee waves show the small amplitude of non-hydraulic jump type with the maximum cross-ridge wind only $20 \mathrm{~ms}^{-1}$ in the first cycle and 40 $\mathrm{ms}^{-1}$ in the second cycle. The potential temperature, streamline and horizontal velocity given by Figures $\mathbf{5}$ and $\mathbf{6}$ show that, in the first cycle stage, the duration of wave systems are shortened greatly and lee waves have dissipated after integrating of 12,000 s. In the second cycle stage, the small amplitude lee waves regenerate above the boundary layer after integrating of $36,000 \mathrm{~s}$, as time increases, the amplitude and intensity of lee waves of the boundary layer aloft increase greatly, accompanied by the rotors and reversal flow in the boundary layer after
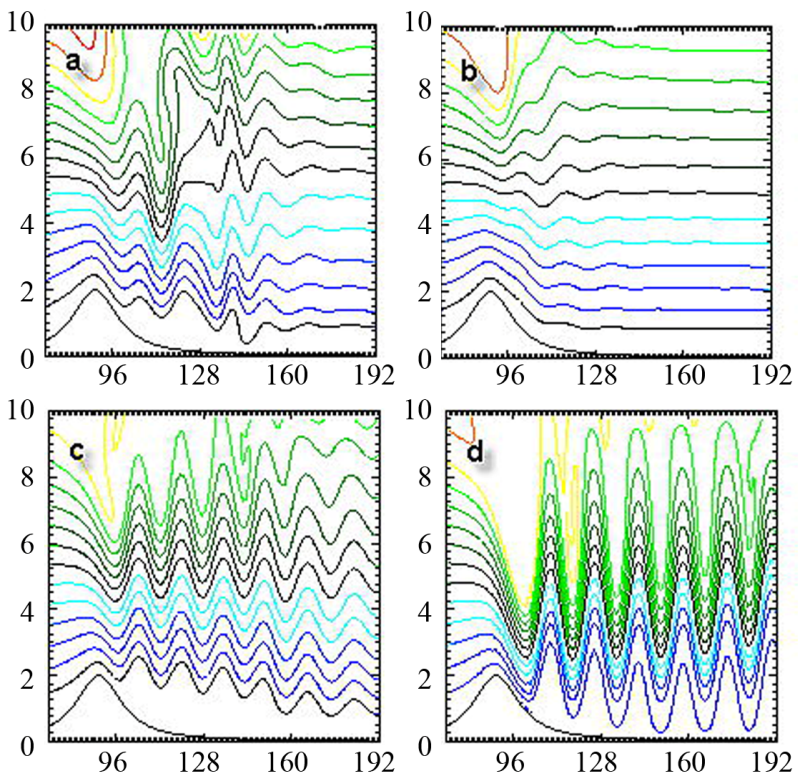

Figure 5. Same as Figure 1 but for the case with surface friction of $C_{d}=10^{-4}$.
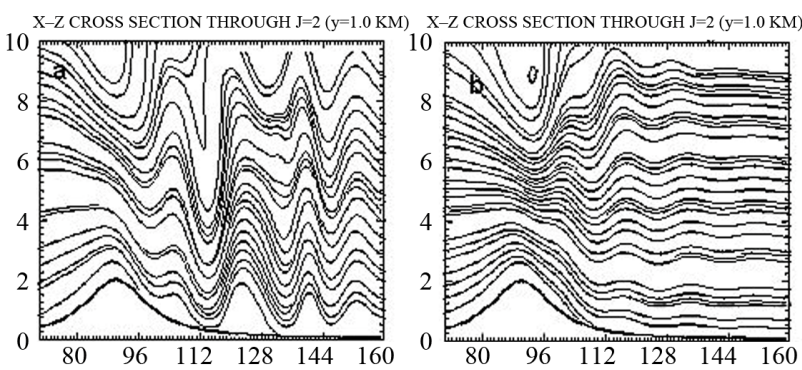

$\mathrm{X}-\mathrm{Z}$ CROSS SECTION THROUGH $\mathrm{J}=2(\mathrm{y}=1.0 \mathrm{KM})$ $\mathrm{X}-\mathrm{Z}$ CROSS SECTION THROUGH $\mathrm{J}=2(\mathrm{y}=1.0 \mathrm{KM})$
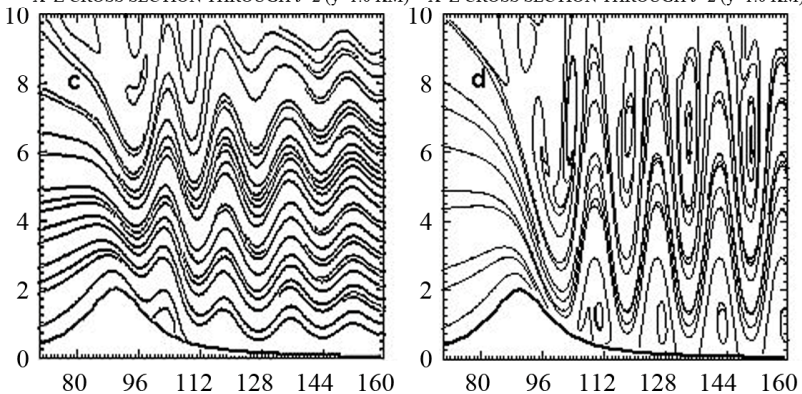

Figure 6. Same as Figure 2 but for the case with surface friction of $C_{d}=10^{-2}$.

integrating of 76,000 s. Finally, the lee waves of nonhydraulic jump type have dissipated after integrating of $126,000 \mathrm{~s}$ and complete the second cycle stage.

\section{Discussion and Conclusion}

The stratified flow over mountain is investigated by the Advanced Regional Prediction System (ARPS) model, the results show that the regeneration of lee wave systems is apparent and allows the wave systems to undergo one transition of two cycles. Most significantly, the ef- 
fect of surface friction on lee wave amplitude and intensity varies with the magnitude of the drag coefficient, especially, cyclic regeneration of lee waves and down slope winds in our runs was found to be extremely sensitive to the magnitude of the drag coefficient. For the typical drag values $C_{d}=10^{-3}$, surface friction promotes the formation of the stationary mountain lee waves and the stationary hydraulic jump, especially, promotes the boundary layer separation, the generation of the low-level turbulent zones and rotor circulation or reversal flow within the boundary layer. When the drag coefficient is smaller $C_{d}=10^{-4}$, the lee waves remain steady states and the first cycle maintains much longer than that of the case $C_{d}=$ $10^{-3}$. In the case of the highest drag coefficient $\left(C_{d}=\right.$ $10^{-2}$ ), surface friction suppresses wave breaking and the onset of hydraulic jump, and reduces greatly the amplitude and intensity of lee waves and down slope wind. It can be concluded that, the interaction of lee waves and the boundary layer process, lee waves induce rotors and rotors induce lee waves, finally determine the characters of lee wave systems.

Extending to the case of three-layer flow over mountain, that is, the static stability of the lower (below 2.5 $\mathrm{km})$, middle layer $(2.5 \mathrm{~km}-5 \mathrm{~km})$ and upper atmosphere (above $5 \mathrm{~km}$ ) are 0.02, 0.01 and 0.015, respectively, and keep the same physical domain and the other parameters as before. The results show that the train of lee waves becomes the dominant feature, and lee waves and rotors show the same characters of cyclic regeneration as the uniformly stratified flow over mountain. However, extending to the mean state critical layer flow over mountain, the base state wind is $20 \mathrm{~ms}^{-1}$ below $5 \mathrm{~km}$ and is reduced from $20 \mathrm{~ms}^{-1}$ at $5 \mathrm{~km}$ to zero above $7 \mathrm{~km}$. It suggests that the critical layer increases the amplitude and intensity of lee waves and surface friction promotes the forming of lee rotors, but the evolution of lee waves is relatively minor as time increases both with and without surface friction. The responses of flow to topography are mainly determined by the factors of the topography, stratification and wind configuration.

\section{REFERENCES}

[1] R. B. Smith, "The Influence of Mountains on the Atmosphere. Advances in Geophysics,” Academic Press, Amsterdam, 1979, pp. 87-230. doi:10.1016/S0065-2687(08)60262-9

[2] R. B. Smith, "Stratified Flow over Topography. Environment Stratified Flows,” Academic Press, Amsterdam, 2002, pp. 121-162.

[3] D. R. Durran, "Mountain Waves and Down-Slope Flows. Atmospheric Processes over Complex Terrain, Metero. Monographs," American Meteorological Society, Vol. 23, No. 45, 1990, pp. 59-81.

[4] P. G. Baines, “Topographic Effects in Stratified Flows,”
Cambridge University Press, Cambridge, 1995, pp. 1-488.

[5] M. G. Wurtele, R. D. Sharman and A. Data, "Atmospheric Lee Waves,” Annual Review of Fluid Mechanics, Vol. 28, 1996, pp. 429-476. doi:10.1146/annurev.fl.28.010196.002241

[6] V. Grubisic and P. K. Smolarkiewicz, "The Effect of Bottom Friction on Shallow-Water Flow Past an Isolated Obstacle,” Journal of the Atmospheric Sciences, Vol. 54, No. 11, 1997, pp. 1943-1960.

[7] G. R. Mamatsashvili, V. R. Avsarkisov, G. D. Chagelishvili, R. G. Chanishvili and M. V. Kalashnik, "Transient Dynamics of Nonsymmetric Perturbations Versus Symmetric Instability in Baroclinic Zonal Shear Flows,” Journal of the Atmospheric Sciences, Vol. 67, No. 9, 2010, pp. 2972-2989. doi:10.1175/2010JAS3313.1

[8] M. E. McIntyre, "Spontaneous Imbalance and Hybrid Vortex Gravity Structures,” Journal of the Atmospheric Sciences, Vol. 66, No. 5, 2009, pp. 1315-1326. doi:10.1175/2008JAS2538.1

[9] R. Plougonven, C. Snyder and F. Zhang, "Comments on Application of the Lighthill-Ford Theory of Spontaneous Imbalance to Clear-Air Turbulence Forecasting,” Journal of the Atmospheric Sciences, Vol. 66, No. 8, 2009, pp. 2506-2510. doi:10.1175/2009JAS3027.1

[10] C. Snyder, R. Plougonven and D. J. Muraki, "Mechanisms for Spontaneous Gravity Wave Generation within a Dipole Vortex," Journal of the Atmospheric Sciences, Vol. 66, No. 11, 2009, pp. 3464-3478. doi:10.1175/2009JAS3147.1

[11] E. Richard, R. Mascart and E. C. Nicherson, "The Role of Surface Friction in Down Slope Windstorms," Journal of Applied Meteorology, Vol. 28, No. 4, 1989, pp. 241-251. doi:10.1175/1520-0450(1989)028<0241:TROSFI $>2.0$.CO ;2

[12] H. Olafsson and P. Bougeault, "The Effect of Rotation and Surface Friction on Orographic Drag,” Journal of the Atmospheric Sciences, Vol. 54, No. 1, 1997, pp. 193-210. doi:10.1175/1520-0469(1997)054<0193:TEORAS $>2.0 . \mathrm{C}$ $\mathrm{O} ; 2$

[13] H. Olafsson and P. Bougeault, "Why Was There No Wave Breaking in PYREX?” Beitraege zur Physik der Atmosphaere, Vol. 70, No. 2, 1997, pp. 167-170.

[14] J. D. Doyle and D. R. Durran, "The Dynamics of Mountain-Wave Induced Rotors,” Journal of the Atmospheric Sciences, Vol. 59, No. 2, 2002, pp. 186-201. doi:10.1175/1520-0469(2002)059<0186:TDOMWI >2.0. $\mathrm{CO} ; 2$

[15] M. S. Peng and W. T. Thompson, "Some Aspects of the Effects of Surface Friction on Flow over Mountains,” Meteorological Society, Vol. 129, No. 593, 2003, pp. 25272557. doi:10.1256/qj.02.06

[16] H. M. Albert, A. Joan and P. Riwal, "On the Intermittency of Gravity Wave Momentum Flux in the Stratosphere," Journal of the Atmospheric Sciences, Vol. 69, No. 11, 2012, pp. 3433-3448. doi:10.1175/JAS-D-12-09.1

[17] J. Knox, D. McCann and P. Williams, "Application of the Lighthill-Ford Theory of Spontaneous Imbalance to ClearAir Turbulence Forecasting," Journal of the Atmospheric 
Sciences, Vol. 65, No. 10, 2008, pp. 3292-3304. doi:10.1175/2008JAS2477.1

[18] F. Lott and R. Plougonven, “Gravity Waves Generated by
Sheared Three-Dimensional Potential Vorticity Anomalies,” Journal of the Atmospheric Sciences, Vol. 69, No. 7, 2012, pp. 2134-2151. doi:10.1175/JAS-D-11-0296.1 